\title{
3D-RTK CAPABILITY OF SINGLE GNSS RECEIVERS
}

\author{
W. Stempfhuber \\ Beuth Hochschule für Technik Berlin, University of Applied Sciences
}

KEY WORDS: Low Cost, GNSS, Single Frequency, Carrier Phase Ambiguity Resolution, 3D-Positioning

\begin{abstract}
:
Small, aerial objects are now being utilised in many areas of civil object capture and monitoring. As a rule, the standard application of a simple GPS receiver with code solutions serves the 3D-positioning of the trajectories or recording positions. Without GPS correction information, these can be calculated at an accuracy of 10-20 metres. Corrected code solutions (DGPS) generally lie in the metre range. A precise 3D-positioning of the UAV (unmanned aerial vehicle) trajectories in the centimetre range provides significant improvements. In addition, the recording time of each sensor can be synchronized with the exact time stamp of the GNSS low-cost system.
\end{abstract}

In recent years, increasing works on positioning from L1 GPS raw data have been published. Along with this, the carrier phase measurements with the established evaluation algorithms are analysed in the post processing method to centimetre-exact positions or to high-precision 3D trajectories [e.g. Schwieger and Gläser, 2005 or Korth and Hofmann 20011]. The use of reference information from local reference stations or a reference network serves the purpose of carrier phase ambiguity resolution. Furthermore, there are many activities worldwide in the area of PPP techniques (Precise Point Positioning). However, dual frequency receivers are primarily used in this instance. Moreover, very long initialisation times must be scheduled for this.

A research project on the subject of low-cost RTK GNSS was developed for real-time applications at the Beuth Hochschule für Technik Berlin University of Applied Sciences [Stempfhuber 2012]. The overall system developed for the purpose of real-time applications with centimetre accuracy is modularly constructed and can be used for various applications (http://prof.beuthhochschule.de/stempfhuber/seite-publikation/). With hardware costing a few hundred Euro and a total weight of 500-800g (including the battery), this system is ideally suited for UAV applications. In addition, the GNSS data processed with the RTK method can be provided in standardised NMEA format. Through the reduced shadowing effects of the aerial objects, GNSS external factors such as multipath cause few problems. With L1 carrier phase analysis, the baseline computation must nevertheless remain limited at the range of a few kilometres. With distances of more than 5 kilometres between the reference station and the rover station position, mistakes arise in the decimetre area.

The overall modular system consists of a low-cost, single-frequency receiver (e.g. uBlox LEA4T or 6T receiver), a L1 antenna (e.g. the Trimble Bullet III), a developed data logger including an integrated WLAN communication module for storage and securing of the raw data as well as a power supply. Optimisation of the L1 antenna has shown that, in this instance, many problems relating to signal reception can be reduced. A calibration of the choke-ring adaptors for various antenna calibration facilities results in good and homogeneous antenna parameters. In this situation, the real-time algorithm from the Open Source project RTKLib [Takasu, 2010] generally runs on a small computer at the reference station. In this case, the data transfer from the L1 receiver to the PC is realisable through a serial cable. The rover station can transfer the raw data to the computing algorithm over a WLAN network or through a data radio. Of course, this computational algorithm can also be adapted to an integrated computing module for L1 carrier phase resolutions. The average time to first fix (TTFF) amounts to a few minutes depending on the satellite constellation. Different test series in movement simulators and in moving objects have shown that a stable, fixed solution is achieved with a normal satellite constellation. A test series with a Microdrones quadrocopter could also be conducted. In comparison of the RTK positions with a geodetic dual frequency receiver, differences are in millimetre ranges. In addition, reference systems (based on total stations) are present for the precise examination of the kinematically captured positioning [Eisenbeiss et al. 2009].

\section{Introduction}

Unmanned aerial vehicles (UAVs) register data capture (as a general rule, aerial images) from a planned flight route. Singlefrequency GPS receivers are primarily used in the course of this. These 3D flight trajectories can be determined from the analysed L1 code observations from about 10-20 metres and with differential code observations from $0,5-3,0$ metres of position accuracy. For DGPS applications, there are many free and purchasable Ground-Based Augmentation Systems (in Germany, e.g., Sapos EPS, Beacon, Egnos, etc.). These processes are clearly listed in Augath et al. 2000. Real-time kinematic GNSS processes are able to capture 3D trajectories centimetre-exactly. For real-time applications in the centimetre range, carrier phase ambiguities must be resolved. This has been conducted in geodesy with multi-frequency receivers for many years. Nevertheless, research projects with low-cost receivers (L1, GPS, single-frequency receivers) show that with normal satellite conditions (minimal shadowing effects, minimisation of disturbing influences and a satellite count of 5-6), centimetreexact 3D positions with static position data [e.g., Glabsch, et al. 2009] as well as with kinematic position data [Stempfhuber, 2011] are achieved on a real-time basis. 3D trajectories with real-time applications, e.g., the navigation of moving objects, are therefore available in standardised NMEA output format 0183 (www.nmea.de). In addition, such a process delivers an absolute time reference for the synchronisation of various measuring sensors and capture sensors. The time impulse noise 
PPS (Pulse per Second Process) amounts to a few $\mu$ in the complete system.

Many geodetic applications require an exact and cost-efficient measuring system for the capture of absolute $2 \mathrm{D}$ or $3 \mathrm{D}$ point coordinates with zenith metre accuracy on a real-time basis. Additionally, the latency periods should only amount to a few milliseconds. Moreover, the complete measuring equipment with many applications should have a low weight and use little power. This is also the case with UAV utilisation (Unmanned Aerial Vehicle). At the Beuth University of Applied Sciences, a GNSS RTK low-cost system (real time kinematic) has been developed and optimised for some years. Various test measurements and field applications have shown that this approach can be also deployed for exact positioning with UAV applications. For the improvement and optimisation of a lowcost GNSS system, it not only makes sense to use a GPS receiver with the $\mathrm{L} 1$ frequency $1,575.42 \mathrm{MHz}$ (e.g., ublox receiver Lea4/6T), but also GLONASS (satellite-dependent frequency of $1598-1605 \mathrm{MHz}$ ) and Galileo receivers with the E1 frequency (identical to the L1 GPS frequency). For example, this boards ublox Lea7N or Navis can process GPS L1 and Glonass L1 frequencies. Aside from the receivers, an algorithm including the hardware communication must be available for the real time analysis of the raw data. Additionally alongside the general GNSS magnitudes of influence, the special factors and occurrences are to be particularly observed with UAV applications. This was and is more precisely processed in various assignments and in a research project (cf. Research Assistance VI at the Beuth University of Applied Sciences). A minimisation of the hardware weight and the intelligent integration of the complete system in the aerial object are further targeted. The stability of the carrier phase ambiguity solution of the baseline analysis must also be intensely analysed for this. The maximum ranges of data transmission between the Rover station and base station as well as the processing unit are to be additionally considered. In this instance, an interferencefree communication between the individual components is to be kept in mind. The storage of the GNSS raw data in standardised RINEX format must be ensured for later data analyses in the post-processing analysis.

The present GNSS RTK Low-cost System at the Beuth University of Applied Sciences consists of a flexible construction. With this, the individual components can be freely adapted and assembled. At the moment, the GNSS reference and Rover unit can be operated with different low-cost boards such as ublox, Sky Traq, NovaTel, Hemisphere and Superstar. The implementation and analysis of other GNSS boards is targeted. Very good results have been achieved in the past with the ublox receivers Lea $4 \mathrm{~T}$ and $6 \mathrm{~T}$ (L1 GPS receiver). New ublox Lea 7 generations also use the L1 frequency of the GLONASS satellites and can also use the E1 frequency of the Galileo satellites. Regional satellite navigation systems such as QZSS or Compass are not considered in this article. The receivers mentioned can be operated with different low-cost antennas and also with geodetic antennas, if necessary. The very light ublox ANN-MS Antenna was little considered in the previous work. Very good results could be achieved with the Trimble Bullet III Antenna, among others. Interference signals could be extensively minimised through its own choke ring construction (figure 1). This antenna construction was assessed in different assignments and calibrated on the calibration facilities of the Berlin Senate Administration and the University of Technology of Dresden [Carius, 2011 or Stempfhuber, 2012]. The homogeneous antenna models demonstrate this approach.
An identical receiver system can be used as a local reference system. In this instance, very good absolute accuracy and nearly $100 \%$ ambiguity resolution is achieved through short baselines. With a cold start, the initialization time (Time to First Fix, TTFF) amounts to a few minutes. With this, the raw data is processed through the company internal data formats in the GNSS data analysis software (with the ublox receiver, a binary format with the name, ubx). The services for RTCM correction signals from GNSS correction services can also be used (in Germany, for the carrier phase solutions with the services of the surveying authorities, SAPOS, Trimble VRSNow, AXIO-Net ascos and Leica SmartNet, distributed over the NTRIP Process). In this instance, the quality and stability of the network solutions based on multi-frequency receivers (RTCM Standard 3.1 in the MAC process) must be separately examined. With this, the baselines should only amount to a few kilometres. Investigations of the influence of the baseline length on the carrier phase solutions with GPS L1 receivers proved that from a distance of approximately $3-5 \mathrm{~km}$, the position accuracy of 1$3 \mathrm{~cm}$ became significantly worse. At the moment, the maximum measuring and analysis frequency of the system with ublox Lea $6 \mathrm{~T}$ receivers amounts to $10 \mathrm{hz}$. The main component at this approach is based on the open source project RTKLib [www.rtklib.com; Takasu, 2009]. The open source platform provides following subsystems:

$\begin{array}{lll}\text { - } & \text { RTKNavi } & \text { Real time kinematic GNSS } \\ & & \text { Algorithm (NMEA export) } \\ \text { - } & \text { RTKPost } & \text { Post-processing algorithm } \\ \text { - } & \text { RTKPlot } & \text { Visualisation } \\ \text { - } & \text { RTKConv } & \text { RINEX converting tool }\end{array}$

RTKNavi delivers real-time positions in the accuracy range of a few centimetres. With RTKPost, the recorded raw data can be post-processed in the format of the above-mentioned receiver or in RINEX format. Playing back the raw data with variable configuration settings of the baseline calculation and position outputs in a serial interface can also be very helpful tools. Another component displays the communication between the receivers and the calculation software. A developed WLAN module establishes a wireless connection to the Rover unit through an ad hoc network. The real-time positions and the time stamp of the measurement are on the processing unit that, in the past, was primarily directly connected to the local reference station with a cable. Ranges of some hundred metres can be achieved through optimised WLAN broadcasting antennas. Another communication possibility arises over a data broadcast modem (e.g., Satelline 3ASx). Data transmission through the mobile Internet (via an Android Smartphone) is currently being developed. The modular construction of the complete system is as follows (weight according to configuration, including battery, approximately 500-800g). 


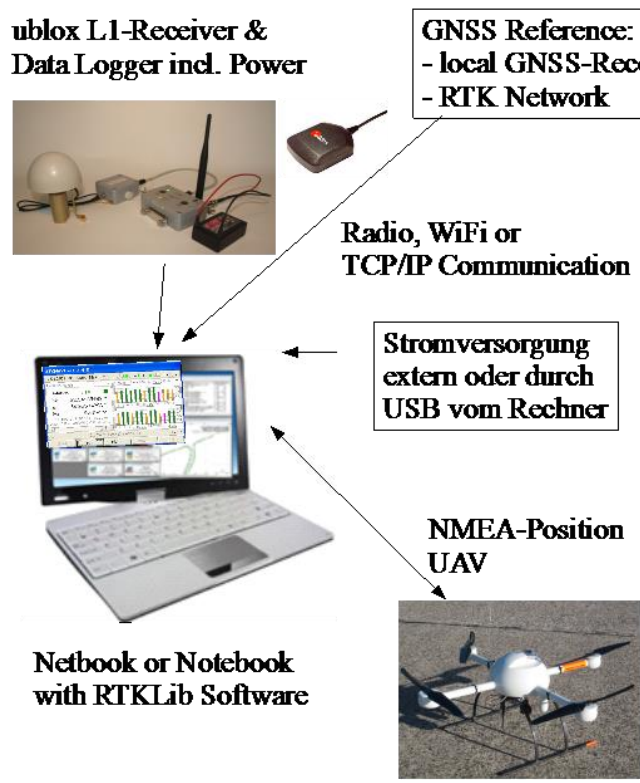

Figure 1, Overall Low-cost RTK GNSS System

\section{RTK Low-cost GNSS Applications}

The L1 low-cost RTK system described in the preceding paragraph is based on a functional platform and can be easily deployed for the most varied of applications. Up to now, the geodetic applications have been in the following areas:

\section{Post-processing}

In this case, low-cost GNSS receivers are particularly wellsuited for the processing of raw data (RINEX data). The data analysis system can conduct the raw data analysis classically as a baseline analysis or with an automated batch file or by introducing the real-time data directly into the data analysis system. The system construction is, among other items, described in [Stempfhuber, 2012]. Moreover, the individual receiver components and the complete system are currently being examined in different dissertations, particularly for interference influence, response time and long-term stability.

\section{Navigation and Machine Control and Guidance}

Up to now, simulations of different tasks by the machine guidance system and for the navigation of precision farming applications have been simulated, recorded and analysed particularly with Mindstorms NXT components. A complete system construction with absolute position comparison between the RTK low-cost system and a reference from a destinationtotal station (cf. Section 4) has been analysed. It is based on the approach of Figure 3 . The first results in a movement simulator were published [Stempfhuber, 2011].
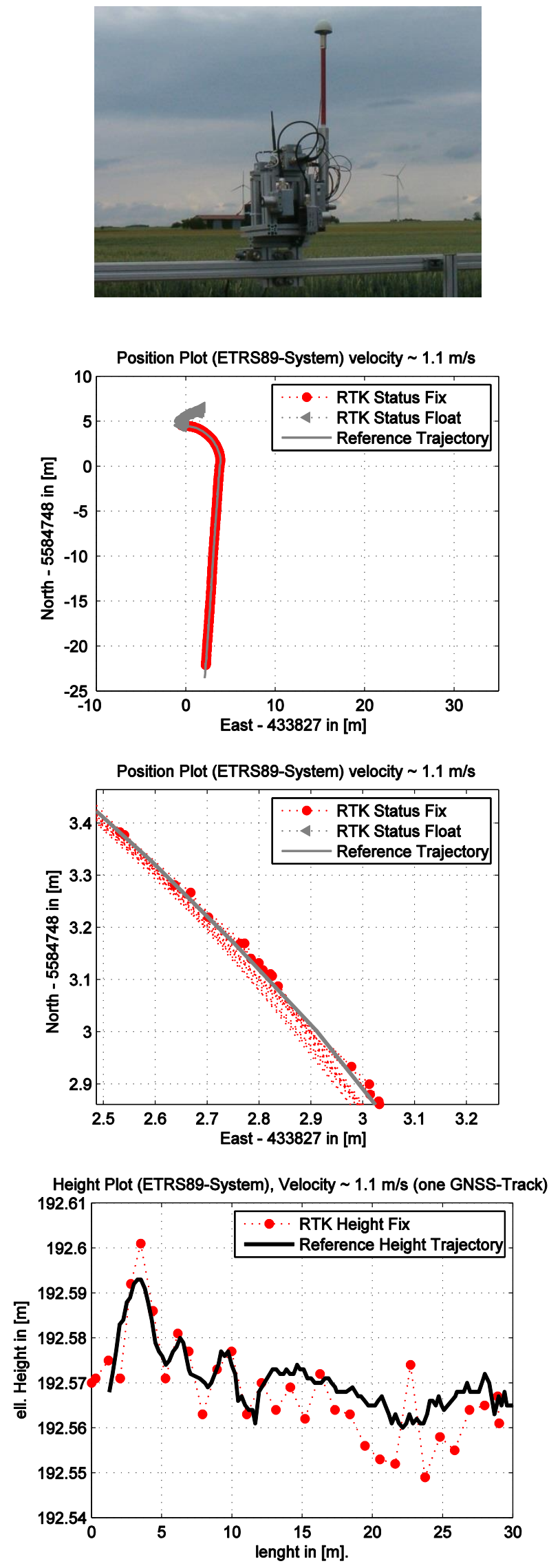

Figure 2, 3D-Navigation with the Low-cost RTK GNSS System at a Reference Track 


\section{UAV Applications}

The first tests were conducted in a Quadrocopter from the firm, Microdrones, at the airfield in Stendal with the firm, GeoMetric (Fig. 3). With this, it was demonstrated that the data broadcast used with the ad hoc WLAN only reaches to approximately $150 \mathrm{~m}$. Nevertheless, the raw data analysis proved a $100 \%$ fixed solution of the carrier phases. Testing was conducted here with the above-mentioned antennas (ANN from ublox and Trimble Bullet III). A position plot shows the highprecision flight trajectory.
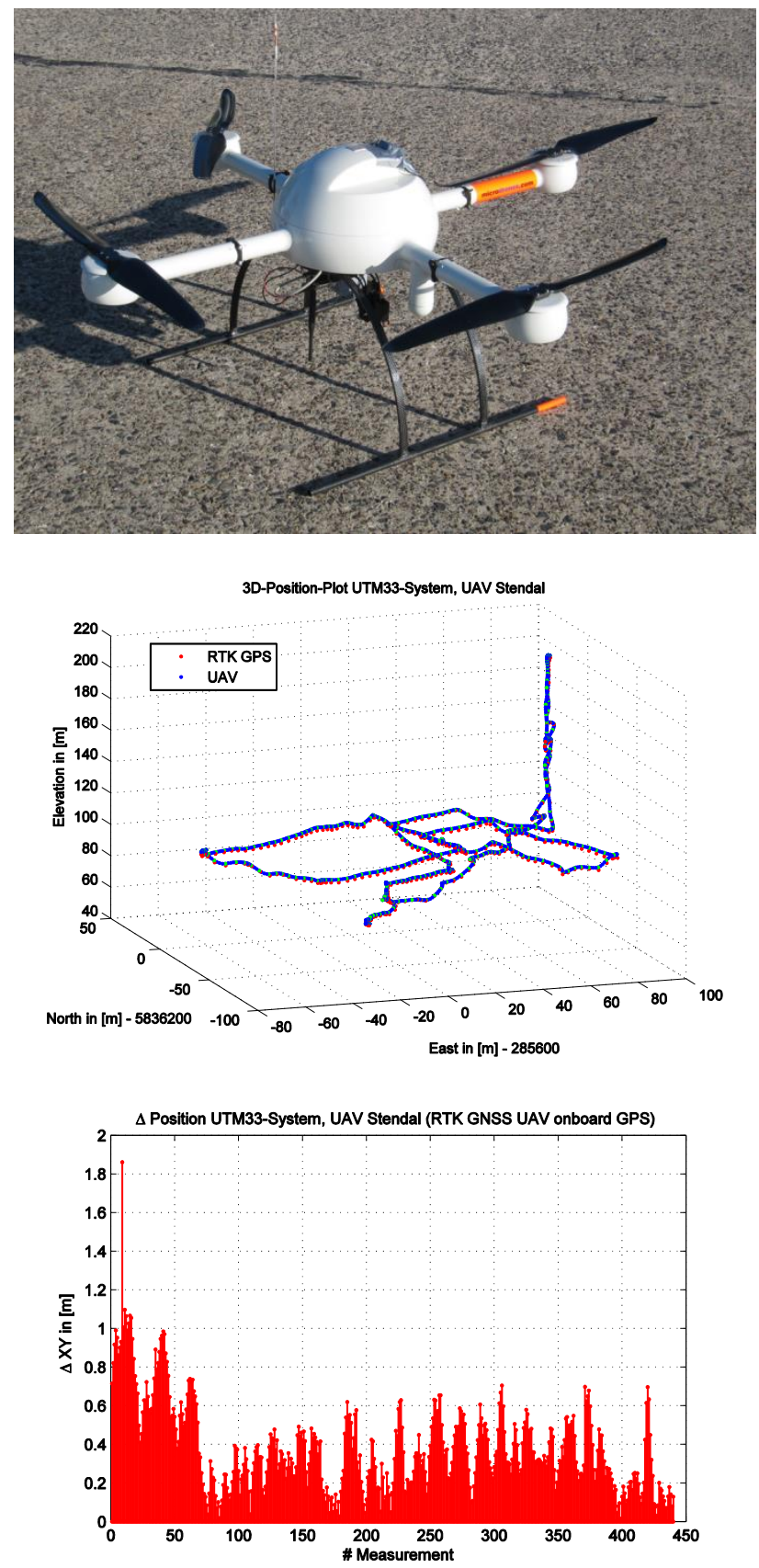

Figure 3, UAV GNSS 3D-Navigation (Comparison: Standard UAV GPS with the L1 RTK GNSS System)

\section{Standard Surveying}

A system is currently being constructed that, based on the described components, facilitates a simple Rover system in point capture and surveying. The approach of the complete system has already delivered the first positive test results. This approach is a reasonable and easy alternative for the capture of pass points in photogrammetric analyses or reference data with centimetre-exact demands on a classic RTK GNSS multifrequency system.

\section{Reference Service e.g.: \\ (Sapos, ascos, Trimble VRSNow, Smart-Net)}

or

\section{Local Reference Station (ublox Lea 4/6T-Empfānger) mit Kabelverbindung zum Rechner}

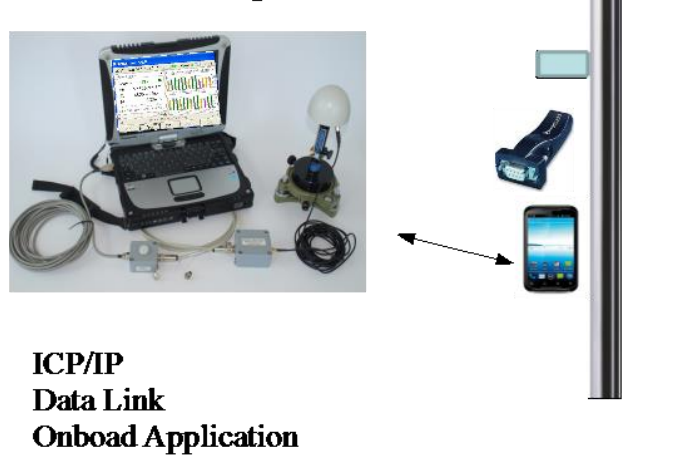

兽

Figure 4, L1 RTK GNSS Rover System, e.g. Ground Control Point Acquisition

For an UAV application of the measuring system, the following subsidiary tasks must still be processed in greater detail:

- $\quad$ precise definition of the UAV 3D trajectory applications

(speed, factors, accelerations, distances to the GNSS reference station)

- Integration of GLONASS receivers

- Integration of the software including the communication

- adjustment of processing parameters and real-time filters

- evaluation of general parameters and the UAV application factors

- $\quad$ analysis of 3D residual

- PPS event for of sensor synchronization

- Optimization of the overall system 


\section{Total Station based Reference Systems}

For the development of an exact GNSS positioning system for UAV applications, a reference system is generally also required. A great deal of scientific work in this subject area has already been conducted (cf. http://prof.beuth-

hochschule.de/stempfhuber/seite-publikation). This reference system is based on an approach suited for the field with total station. In this instance, the capture of the position data with the total station must be particularly optimised. Publications with Leica [Kirschner and Stempfhuber, 2008] and Trimble total station [Stempfhuber, 2009] describe the technical capacities. These can be easily introduced in research groups. Here, the 3 trajectories are determined in the range of a few millimetres. A publication on UAV applications describes the method and initial results with a GPS stand-alone system [Eisenbeiss et al. 2009]. A possible system structure is outlined in Figure 5. The integration of a precise time reference is usually difficult since the exact time of measurement on the tachymeter can only be determined by approximately 50 to $100 \mathrm{~ms}$.
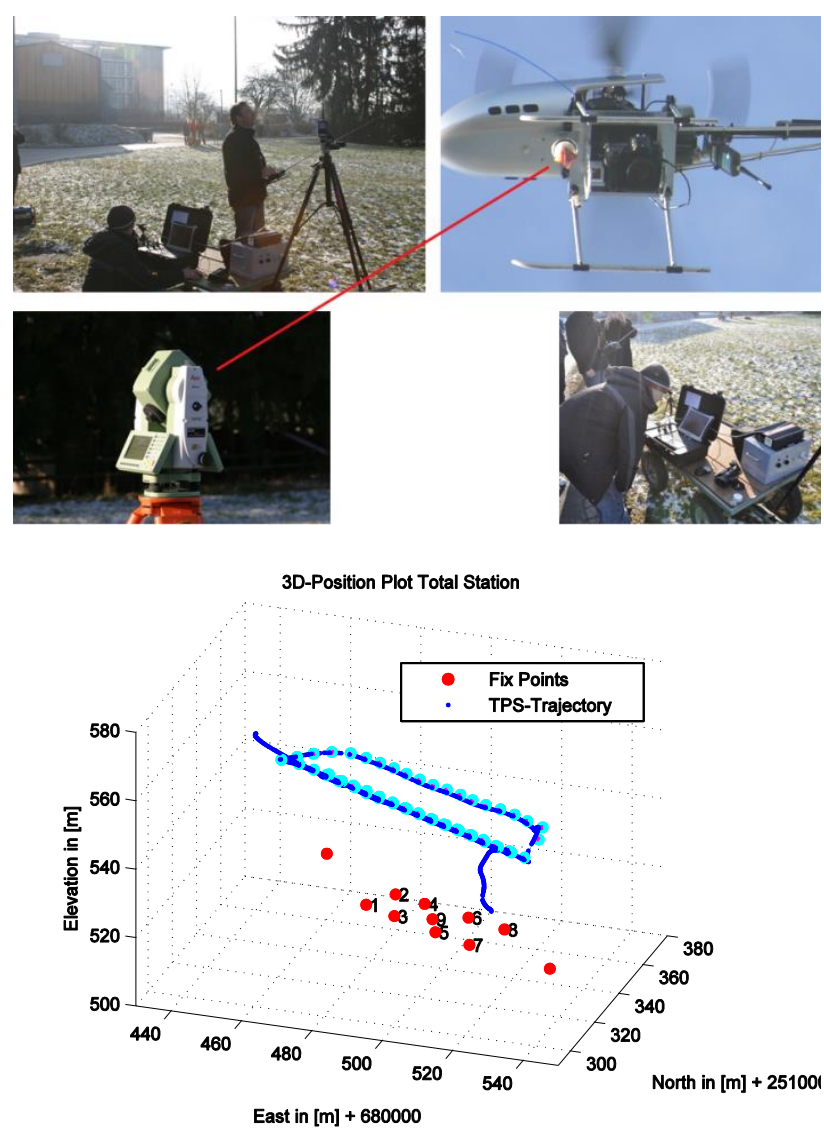

Figure 5, UAV 3D Reference System (Total Station)

\section{Summary and Outlook}

The approach of the described complete system and the first UAV test measurements have demonstrated that such a RTK low-cost GNSS system can easily carry out an exact positioning. A research proposal is currently being developed in cooperation with various partners. The system components are being further developed and evaluated in various tasks. In addition, integration into a mobile unit is being pursued. Here, the RTKLib Project would need to be transferred to a different software platform. In addition, the enhancement with GLONASS and Galileo measurements is to be precisely considered. The algorithm and the ublox LEA 7 receiver allow for such an approach. Furthermore, a stable communication over long distances for RTK applications must be optimised and tested. The approach via a TCP/IP transfer with a Smartphone is promising here.

Analyses with the described L1 RTK GNSS system in a reference line proved the following results. 
Augath, W., Lechner, W., Peters, R. (2000): Combination of DGPS and LORAN-C for Users on the 1-3 m-Level. DGONSymposium 2000, Bonn.

Carius R.: Untersuchungen zur Kalibrierung von GPSAntennen, Diplomarbeit an der Beuth Hochschule für Technik Berlin, unveröffentlicht 2011.

Glabsch J., Heunecke O. und Schuhbäck S.: Hangüberwachungen mittels Low Cost GNSS im alpinen Raum - Ansätze und Erfahrungen, ETH Zürich, AHORH 2009.

Eisenbeiss, H. Stempfhuber, W. and Kolb, M.: Genauigkeitsanalyse der 3D-Trajektorie von Mini-UAVs, Tagungsband der Deutschen Gesellschaft für Photogrammetrie und Fernerkundung (DGPF) 18/2009.

Kirschner H. and Stempfhuber W., The Kinematic Potential of Modern Tracking Total Stations - A State of the Art Report on the Leica TPS1200+, In: Stempfhuber, W and Ingensand, H. [Eds.], MCG-Proceedings, pp. 51-60, 2008.

Korth, W. und Hofmann, U.: Softwareentwicklung für Positionsbestimmung mit Satelliten, Abschlussbericht Forschungsassistenz VI, www.beuth-hochschule.de, 2011.

Schwieger, V. and Gläser, A.: Possibilities of Low Cost GPS Technology for Precise Geodetic Applications. Proceedings on FIG Working Week 2005, Kairo, 2005.

Stempfhuber W.: Verification of the Trimble Universal Total Station (UTS) Performance for Kinematic Applications, In: Grün/Kahmen (Eds), Optical 3-D Measurement Techniques IX, 2009, Vienna, FIG Commission 5 and 6, IAG Special Commission 4, ISPRS Commission 5, pp. 211-221, 2009.

Stempfhuber, W. and Buchholz; M.: A Precise, Low-Cost RTK GNSS System for UAV Applications, ISPRS Archives XXXVIII-1/C22 with ISSN Number: 1682-1777, 2011. Stempfhuber W. und Alberding J.: Geodätische Monitoringsysteme mit RTK Low-Cost-GNSS, AVN 4/2012, VDE Verlag GmbH, 2012.

Stempfhuber, W., Leistungsfähigkeit von Low-Cost RTK GNSS-Systemen zur genauen Positionsbestimmung von UAVAnwendungen, 19. Workshop Computer-Bildanalyse in der Landwirtschaft/2. Workshop Unbemannte autonom fliegende Systeme (UAS) in der Landwirtschaft, Bornimer Agrartechnische Berichte Heft 81, ISSN 0947-7314, 2013.

Takasu, T.: RTKLIB: Open Source Program Package for RTKGPS, FOSS4G 2009 Tokyo, Japan, November 2, 2009. 